\title{
Prevalence of mental disorders in the general population referring to a medical educational center in Rasht, Iran
}

\author{
Yousefzadeh Sh, $\mathrm{PhD}^{1}$, Pilafkan J, MSc${ }^{2}$, Rouhi-Balasi L, MSc ${ }^{3 *}$, Hosseinpour M, MSc ${ }^{4}$, Khodadady \\ $\mathrm{N}, \mathrm{PhD}^{5}$ \\ 1- Professor, Dept., of Neurosurgery, Guilan Road Traffic Research Center, Guilan University of Medical Sciences, Rasht, \\ Iran. 2- MSc in Cilinical Psychology, Poursina Medical Educational Center, Guilan University of Medical Science, Rasht, \\ Iran. 3- Instructor, Dept., of Medical Surgical Nursing, Guilan Interventional Cardiovascular Research Center, Heshmat \\ Hospital, Guilan University of Medical Sciences, Rasht, Iran. 4- MSc in Phisiology, Guilan Road Traffic Research Center, \\ Guilan University of Medical Sciences, Rasht, Iran. 5- PhD Candidate of Nursing, Guilan Road Traffic Research Center, \\ Guilan University of Medical Sciences, Rasht, Iran.
}

Received: June 2015, Accepted: August 2015

Background: Mental disorders in patients with chronic medical disorders are associated with, performance degradation, poor quality of life, lack of cooperation with treatment and inappropriate use of medical services, and increased costs. The objective of this study was to determine the prevalence of mental disorders in the general population referring to an educational medical hospital in Rasht, Iran, in 2013.

Materials and Methods: In this cross-sectional study, 1725 patients who referred to a medical educational center in Rasht were selected using convenience sampling method. Data were collected through clinical interviews and using inpatients' psychological condition analysis form. Data were analyzed using descriptive and inferential statistics.

Results: Results showed that the prevalence of mental disorders in this population was $48.6 \%$. The statistical test results showed age $(\mathrm{P}<0.0001)$, marital status $(\mathrm{P}<0.0001)$, occupation, history of substance abuse $(\mathrm{P}<0.0001)$, psychiatric history $(\mathrm{P}<0.0001)$, and a history of suicide attempt $(\mathrm{P}<$ $0.001)$ had significant relationships with presence of mental disorders.

Conclusions: Given the high prevalence of mental disorders in this community, it is recommended that consultation and therapeutic services be provided in health centers for patients' health.

Keywords: Prevalence, Mental Disorders, Patients, Hospital

\section{Introduction}

Today, in most countries in the world, the attempt to become as industrialized as possible, and the rapidly growing population, urbanization, and migration have resulted in an increase in socio-psychological stresses and nuisances of the societies. These fundamental transformation will also lead to major changes in disease epidemiology and individuals' health requirements (1). In developing countries, which contain $4 / 5$ of the world population, non-contagious diseases like mental disorders are quickly replacing infectious diseases and are the major cause of disability and early deaths. These changes will cause many difficulties for the health systems in these countries (2).

The World Health Organization (WHO) and Harvard researchers have predicted that in 2020, depression disorder will be the $2^{\text {nd }}$ reason of disability instead of the $4^{\text {th }}$. These predictions indicate that the proportion of psychological and neurological disorders will increase about $50 \%$ from their current $11 \%$ of

\footnotetext{
*Corresponding author: Leila Rouhi-Balasi, Dept., of Medical Surgical Nursing, Guilan Interventional Cardiovascular Research Center, Heshmat Hospital, Guilan University of Medical Sciences, Rasht, Iran. Email: roohi_balasi@yahoo.com
} 
the world diseases load and reach around 15\% in 2020 (1).

A mental disorder or disease is a behavioral and psychological pattern occurring in an individual and accompanies disturbance in functioning due to biological, social, psychological, genetic, physical, or chemical disorders. Psychological disorder is measured in terms of normal range deviation intensity (3, 4). Generally, Psychological disorders affect a high percentage of the population and are one of the major reasons behind deaths and disability $(5,6)$. Psychological disorders are considered as a serious and common problem forming about $20 \%$ of all referrals to healthmedical centers worldwide (7). Contrary to that expressed in previous studies, psychological symptoms are not only experienced by patients with psychological disorders, but also by a remarkable part of the healthy population (8).

There is much evidence showing psychosis symptoms may appear in milder forms in the general population $(5,9)$. A meta-analytical research recently performed by Van os et al. reported the $5-8 \%$ prevalence of such symptoms in the general population (8). Psychological disorders in patients suffering from chronic medical disorders are accompanied with damaged function, bad quality of life, noncompliance with treatment and inappropriate use of medical services, and rising costs (10-14). Epidemiological studies of psychological disorders conducted in Iran all imply this disorder prevalence variability (1-4, $7,10,15,16)$. Based on the obtained results, the prevalence rate of psychological disorders in Kashan, Iran, has been 29\% (3). In the study by Sadjadi et al., the prevalence of psychological disorders among diabetic sufferers was $43.75 \%$ (9). The most prevalent of these disorders were adjustment disorders (12 people), major depression (11 people), generalized anxiety disorder (GAD) (5 people) and panic (3\%) (9). In the research by Mohammadi et al., the prevalence of various psychological disorders in Lorestan Province,
Iran, were $19.05 \%$ (15). The most common psychological disorder group in this study was anxiety disorder $(12.46 \%)(15)$. The results of the study by Hassanshahi et al. also indicated that $34 \%$ of the study subjects in Arsanjan city, Iran, suffer from different psychological disorders (2). In the study by Davasaz et al., the prevalence of psychological disorders in the study subjects in Khuzestan Province, Iran, was reported as $28.7 \%$ (7). In the study by Raeeson et al., based on the Global Severity Index (GSI), $45 \%$ of the study subjects were normal, $47.9 \%$ had significant problems, and $7.1 \%$ had serious psychological disorders (4). In the research by Khosravi, psychological disorders prevalence was 18.33 in the urban and rural population above 15 years of age in Borujen, Iran, and the most common disorders in this population were depression and anxiety disorders (16). Overview of epidemiologic studies of psychological disorders in different countries such as Iran has revealed that there have been very different estimations of the prevalence of these disorders due to the variety of applied tools, sampling method, interview techniques, and diagnostic classification $(3,9$, 15).

The co-occurrence of physical illnesses and mental disorders results in weaker prognosis, use of more resources, higher costs, disability, and poor treatment follow-up (5). Regarding the mentioned cases, their heavy treatment and care costs, and physical and emotional supports by the patients' families and their economic burden should not be ignored (3). This issue, disorder prevention planning, treatment, and basic information on the prevalence of this disorder are of great importance. Moreover, no study has been conducted with this objective. Therefore, according the role of culture and geography in this problem, this study has been conducted with the goal to determine psychological disorders' prevalence in the general population referring to one of the medical-educational centers in Rasht, Iran. 


\section{Materials and Methods}

In this cross-sectional study, the study population consisted of all patients referring to one of the medical-educational centers in Rasht (including all referrals to the hospitalization wards). The inclusion criteria consisted of having a history of psychological disorders, long term hospitalization, lost a family member due to an accident, history of drug abuse, hospitalization after a fight and conflict, history of self-mutilation or suicide, presurgery stress, and posttraumatic stress disorder symptoms. Subjects were selected through convenient sampling method from early to late 2013. As a result, 1725 subjects were included in the research. Oral consents were obtained from the subjects. Then, data were collected through clinical interviews by a clinical psychology master and using the inpatients' psychological condition analysis form. The collected data were analyzed using descriptive statistics (frequency, percentage, mean, and standard deviation) and inferential statistics (chi-square and Fisher's exact tests), and SPSS software (version 16, SPSS Inc., Chicago, IL, USA). In addition, for all inferential tests, all $\mathrm{P}$ values of less than 0.05 were considered significant.

\section{Results}

The mean age of the study population was $42.61 \pm 1.9$. The majority of the subjects were male $(63.8 \%)$ and married $(63.2 \%)$. Most of them were under-diploma holders $(47.7 \%)$, housewives $(30 \%)$, and had no history of drug abuse $(77.3 \%)$ or suicide (96.3\%). Furthermore, the majority of the subjects declared that they had no history of psychological disorder (74.4\%). Accidents were the leading cause of hospitalization. The study findings implied that $48.6 \%$ of the study samples had mental disorders and $51.4 \%$ were psychologically healthy. Furthermore, among the different disorders, mood disorder (23.2\%) had the highest frequency and psychotic disorder had the lowest frequency (1.6\%).
In addition, analyzing the relationship between the demographic factors and the presence of mental disorders in the study subjects has suggested a significant statistical relationship between age and mental disorders ( $\mathrm{P}<$ $0.0001)$. The highest frequency of mental disorders was observed in the age group of 2640 years. In addition, chi-square test revealed a significant relationship between marital status and the presence of mental disorder ( $\mathrm{P}<$ 0.0001) and the highest frequency existed among married individuals. The results indicated a higher prevalence of mental disorders in housewives compared to other groups and this difference was statistically meaningful $(\mathrm{P}<0.0001)$. Moreover, Fisher's exact test results displayed a significant relationship between drug abuse and the existence of mental disorders $(\mathrm{P}<0.0001)$. The majority of the subjects with drug abuse had a mental disorder. In this study, all the individuals with a history of suicide had one mental disorder and the relationship between these two variables was statistically significant $(\mathrm{P}<0.0001)$. Moreover, the majority of those who had a history of psychiatric disorder had one mental disorder and this relationship was significant $(\mathrm{P}<0.0001)$. Nevertheless, in this research, no significant relationship was observed between gender and education level, and mental disorders.

\section{Discussion}

The results of the present study depicted that about half of the subjects had one mental disorder and the highest frequency was that of mood disorders. In various studies, different results have been observed in this respect. In the research by Salari et al., the prevalence of psychological problems was estimated as $17.5 \%$ (1), in the study by Ahmadvand et al. as $29 \%$ (3), and in the research by davasazirani as $28.7 \%$ (7). These differences can be due to the differences in tools applied, sampling method, and more importantly cultural, economic, social aspects and the study population's age group. 
In the current research, the maximum prevalence of psychological disorders was observed in the age group of 26-40 years. This finding was the same as that of the study by Mohammadi et al. (15) and Khosravi et al. (16). However, this finding is inconsistent with that of the research by Salari et al. (1) and Hassanshahi et al. (2). It seems that this age period is the peak of socioeconomic activities of the individuals, and thus, life pressures have probably been effective in the prevalence of psychological disorders in this group.

In addition, it was found that married people suffer from psychological disorders more than other groups (single, divorced, and widowed) the difference of which was statistically significant. The results of the study by davasazirani et al. also indicated the higher prevalence of psychological problems in divorced and married individuals compared to the single individuals (7). However, the results of the study by Salari et al. demonstrated that the probability of affliction with psychological disorders in divorced and widowed individuals is higher than single and married individuals (1). The higher prevalence of mental disorders in married people can be attributed to their heavier familial responsibilities and life pressures.

The highest frequency of mental disorders was among housewives and about half of the retired participants suffered from such disorders. The study findings by Mohammadi reflect the higher prevalence of psychological disorders in retired people compared to others. This finding was confirmed by other studies conducted in Iran and the world. Not having an income, the stressful factor of disability, limited social relationships, and monotonous life may be the factors which cause an increase in mental disorders in retired people (15). In the research by Hassanshahi et al., the maximum rate of psychological disorders was observed among employees (2). The reason for the high prevalence among housewives could be being permanently busy in the family and not having effective relationships with others (16). Moreover, the probable reasons for these problems are low income, stress due to unemployment, limitations in social relationships, and the monotony of life in unemployed individuals and housekeepers (17).

Based on the results, the majority of the participants with drug abuse had mental disorders. This finding was similar to that of the study by Jones et al. (18). This can be the result of substances' effects on these people's nervous system and problems rooted in addiction such as being rejected by family and society, loss of job, and economic issues. Furthermore, Raiisi et al. found that the mental health of non-addicted people was superior to addicted individuals (19). It seems that these findings can be due to high stress load and anxiety.

In addition, the majority of subjects with a history of psychiatric disorders and suicide had one psychological disorder and this relationship was statistically significant. In the study by Sheikholeslami et al., mean total score and subscales scores on the General Health Questionnaire (GHQ) were significantly higher in patients with a history of previous Suicide attempt (20). Thus, it seems that psychological follow-ups have not been conducted appropriately in these individuals and this issue illustrates the necessity of paying attention to such individuals.

\section{Conclusion}

Considering the high prevalence of mental disorders in this society, the creation of a counseling and treatment services system in hospital based centers is recommended in order to promote health in the society. In addition, by screening and offering counseling services in hospitals and the society as follow-up we can take a step to enhance psychological health within the society. Since this study was carried out on inpatients of one hospital, one of the limitations of this research is the physical conditions of the study units that may have affected our findings. 


\section{Acknowledgement}

The researchers sincerely appreciate the cooperation of the Guilan Road Trauma Research Center, Iran, and all the patients who participated in this research.

\section{Conflict of interest: None declared}

\section{References}

1. Salari H, Ghanbari MR, Taziki SA, Padash L, Yousefi MR, LotfaliNejad E. Survey of 15 Years and Older Women's Mental Health Status in Urban Population of Gorgan. Journal of Research Development in Nursing \& Midwifery 2013; 10(1):77-83.

2. Hassanshahi MM, Goodarzi MA, Mirjafari SA, Farnam F. Pervalence of psychiatric disorders in the city Arsanjan. The Quarterly Journal of Fundamentals of Mental Health 2003-2004; 5(19-20):67-83.

3. Ahmadvand A, Sepehrmanesh Z, Ghoreyshi F, Assarian F, Moosavi G, Saee R, et al. Prevalence of mental disorders in general population of Kashan City. Iranian Journal of Epidemiology 2010; 6(2):16-24.

4. Rieson M, Miri M, Dastjerdi R, Sharifzadeh G. Prevalence of mental disorders in Birjand-2010. Journal of Birjand University of Medical Sciences 2012; 19(1):81-7.

5. Gili M, Comas A, García-García M, Monzón S, Antoni SB, Roca M. Comorbidity between common mental disorders and chronic somatic diseases in primary care patients. Gen Hosp Psychiatry 2010; 32(3):240-5.

6. Merikangas KR, Nakamura EF, Kessler RC. Epidemiology of mental disorders in children and adolescents. Dialogues Clin Neurosci 2009; 11(1):7-20.

7. Davasazirani R, Bagheriyazdi SA, Keykhani B, Pakseresht S, Karimi M. Prevalence of Mental Disorders in Khuzestan Province Rural Areas. Iraninan Journal of Psychiatry and Clinical Psychology 2006; 12(1):71-4.

8. Kelleher I, Cannon M. Psychotic-like experiences in the general population: characterizing a high-risk group for psychosis. Psychol Med 2011; 41(1):1-6.

9. Wiles NJ, Zammit S, Bebbington P, Singleton N, Meltzer H, Lewis G. Self-reported psychotic symptoms in the general population Results from the longitudinal study of the British National Psychiatric Morbidity Survey. The
British Journal of Psychiatry 2006; 188(6):51926.

10. Sajjadi A, Bakhshani NM, Baghban-Haghighi M, Samadi R, Lashkaripoor K, Safarzai M. Prevalence of Psychiatric Disorders in Patients with Diabetes Type 2. Zahedan Journal of Research in Medical Sciences 2012; 14(1):825.

11. Ansseau M, Dierick M, Buntinkx F, Cnockaert P, De Smedt J, Van Den Haute M, et al. High prevalence of mental disorders in primary care. J Affect Disord 2004; 78(1):49-55.

12. Cole MG, Bellavance F, Mansour A. Prognosis of depression in elderly community and primary care populations: a systematic review and meta-analysis. Am J Psychiatry 1999; 156(8):1182-9.

13. Forsman AK, Nyqvist F, Wahlbeck K. Cognitive components of social capital and mental health status among older adults: A population-based cross-sectional study. Scand J Public Health 2011; 39(7):757-65.

14. Goldberg SE, Whittamore KH, Harwood RH, Bradshaw LE, Gladman JR, Jones RG, et al. The prevalence of mental health problems among older adults admitted as an emergency to a general hospital. Age Ageing 2011; 41(1):80-6.

15. Mohammadi MR, Davidian H, Noorbala AA, Malekafzali H, Naghavi HR, Pouretemad HR, et al. An epidemiological survey of psychiatric disorders in Iran. Clin Pract Epidemiol Ment Health 2005; 1:16.

16. Khosravi S. Epidemiology of mental disorders of over 15 years populationof in urban and rural of Borojen. Journal of Shahrekord University of Medical Sciences 2003; 4(4):319.

17. Ahmanvand A, Sepehrmanesh Z, Ghoreishi FS, Afshinnejad S. Prevalence of psychiateric disorders in the general population of Kashn in Iran. Arch Iran Med 2012; 15(4):205-9.

18. Jones DR, Macias C, Barreira PJ, Fisher WH, Hargreaves WA, Harding CM. Prevalence, Severity, and Co-occurrence of Chronic Physical Health Problems of Persons With Serious Mental Illness. Psychiatr Serv 2004; 55(11):1250-7.

19. Raiisi F, Anisi J, Yazdi S, Zamani M, Rashidi S. Mental health and child-rearing styles between candidate adult for addiction withdrawal in comparison with non-addict adults. Journal of Behavorial Sciences 2008; 2(1):33-41.

20. Sheikholeslami H, Kani K, Ziaee A. Survey of Precipitating Factors of Suicide Attempts in Persons Who Referred to Emergency Department. Journal of Guilan University of Medical Sciences 2008;17(65):77-87. 